Service social

\title{
La fonction de liaison en organisation communautaire
}

\section{René Lachapelle}

Volume 60, numéro 2, 2014

URI : https://id.erudit.org/iderudit/1027995ar

DOI : https://doi.org/10.7202/1027995ar

Aller au sommaire du numéro

Éditeur(s)

École de service social de l’Université Laval

ISSN

1708-1734 (numérique)

Découvrir la revue

Citer cet article

Lachapelle, R. (2014). La fonction de liaison en organisation communautaire. Service social, 60(2), 115-129. https://doi.org/10.7202/1027995ar

\section{Résumé de l'article}

Les organisateurs et organisatrices communautaires (OC) exercent une fonction de liaison qui s'est transformée au fil des diverses périodes de mise en oeuvre de l'approche communautaire et territoriale depuis les CLSC (Centres locaux de service communautaire) jusqu'aux Centres de santé et de services sociaux (CSSS). Avec la multiplication des approches intersectorielles et des métiers du développement dont les pratiques relèvent aussi de l'organisation communautaire, cette fonction s'est aussi diversifiée. Les fonctions, dont la liaison, constituent un angle mort de la littérature québécoise et nord-américaine sur l'organisation communautaire. La recherche a mis en évidence l'importance du contexte politique dans lequel est mise en oeuvre la fonction de liaison et permis de montrer qu'elle repose sur l'exercice de compétences pratiques : connaître le milieu, mobiliser les acteurs, garantir la cohésion du système local d'action, démocratiser les rapports au pouvoir et rendre accessibles les ressources. 


\title{
La fonction de liaison en organisation communautaire
}

\author{
LACHAPELLE, René \\ Professionnel de recherche \\ Chaire de recherche du Canada en organisation communautaire \\ Université du Québec en Outaouais
}

\section{RÉSUMÉ}

Les organisateurs et organisatrices communautaires $(\mathrm{OC})$ exercent une fonction de liaison qui s'est transformée au fil des diverses périodes de mise en œuvre de l'approche communautaire et territoriale depuis les CLSC (Centres locaux de service communautaire) jusqu'aux Centres de santé et de services sociaux (CSSS). Avec la multiplication des approches intersectorielles et des métiers du développement dont les pratiques relèvent aussi de l'organisation communautaire, cette fonction s'est aussi diversifiée. Les fonctions, dont la liaison, constituent un angle mort de la littérature québécoise et nord-américaine sur l'organisation communautaire. La recherche a mis en évidence l'importance du contexte politique dans lequel est mise en œuvre la fonction de liaison et permis de montrer qu'elle repose sur l'exercice de compétences pratiques: connaître le milieu, mobiliser les acteurs, garantir la cohésion du système local d'action, démocratiser les rapports au pouvoir et rendre accessibles les ressources.

Mots clés : Organisation communautaire, liaison, Centres de santé et de services sociaux, systèmes locaux d'action, mobilisation.

\begin{abstract}
Community organizers (OC) implement a liaison function that has changed according to the various periods of development of the community and territory approach from Local Community Service Centres (CLSC) to Health and Social Services Centres (CSSS). In a context of growing importance of intersectoral approaches and increasing number of development jobs related to community organizing, the function has diversified. Functions, including liaison, are a blind spot in Quebec's and North America's literature about community organizing. The research showed the importance of political context in liaison function practices that are based on five skills: knowing the community, mobilizing stakeholders, ensuring the cohesion of the local action system, democratizing relations with authorities, and making resources accessible for the implementation of actions.
\end{abstract}

Key words: Community organization, liaison, Health and Social Services Centres, local action system, mobilization. 


\section{INTRODUCTION}

L'organisation communautaire est une base commune de professions dont les pratiques visent à renforcer la capacité d'agir de celles et ceux qui sont loin du pouvoir économique ou politique : organisateurs et organisatrices communautaires (OC) en Centres de santé et de services sociaux (CSSS), agents de développement rural et autres métiers du développement qui se sont multipliés à la faveur du développement de programmes publics et privés. Ce sont des pratiques qui doivent composer avec de multiples variables sociales, institutionnelles et conjoncturelles, mais surtout avec des personnes et des organismes autonomes en interaction dans des milieux qui ont leur culture propre. Les OC agissent comme des passeurs entre les acteurs collectifs. L'article présente les résultats d'une recherche menée dans le cadre d'une thèse de doctorat qui voulait répondre à la question suivante : comment des OC pratiquent-ils la fonction de liaison dans le contexte actuel des CSSS et de l'action collective dans les communautés locales du Québec ? Tout en visant plus spécifiquement les conditions de mise en œuvre dans les CSSS, la collecte s'est enrichie de données venant d'autres praticiennes et praticiens du développement qui exercent aussi une fonction de liaison.

Une fonction, selon le Robert, c'est « ce que doit accomplir une personne pour jouer son rôle dans la société, dans un groupe social ». Le concept renvoie donc à ce qui, dans la pratique professionnelle de l'organisation communautaire, permet de produire des résultats. La fonction de liaison caractérise une pratique professionnelle qui «contribue à ce que les individus d'un groupe améliorent leur connaissance, leur compréhension et leurs contacts concernant les individus [d'un] autre groupe et inversement » (Liedholm et Lindberg, 2007, p. 85).

Introduite dans le réseau public de la santé et des services sociaux depuis un peu plus d'une quarantaine d'années, l'organisation communautaire a connu un parcours qui a favorisé son institutionnalisation comme pratique professionnelle. Ce processus historique a modifié les conditions d'exercice et les modes d'action des OC dans la mise en œuvre de la fonction de liaison.

L'article présente les conclusions de la recherche en contextualisant ces pratiques depuis leur développement dans les Centres locaux de services communautaires (CLSC) jusque dans les CSSS. Après une courte mise en contexte du questionnement, la seconde section de l'article présente brièvement le cadre conceptuel et la méthodologie de la recherche. Les sections trois et quatre résument l'essentiel des résultats quant aux transformations de la fonction de liaison dans le temps et sur la base des systèmes locaux d'action (SLA) où elle s'exerce. La section cinq propose une définition de la fonction de liaison telle qu'elle se présente sur le terrain. La conclusion indique quelques conditions de mise en œuvre dans le contexte actuel.

\section{La problématique de départ}

La présence des OC dans les CLSC a suscité depuis le début des questionnements sur leurs rapports, d'une part, avec une institution relevant de l'État et, d'autre part, avec le milieu et les organismes autonomes de la société civile. Au moment où l'État crée ces établissements, « récupérant » les cliniques populaires, selon les uns (Doré et Larose, 1979 ; Gulati et Guest, 1990), ou mettant en place une nouvelle approche de la santé, selon les autres (Jobin, 1986 ; 
Sénéchal, 1994), les allégeances des OC occupent une place centrale dans les analyses que l'on fait de leurs pratiques. Ce qui est significatif de ces questionnements, c'est qu'ils indiquent que l'organisation communautaire en CLSC est en position frontière entre l'institution publique et l'action citoyenne. En durcissant volontairement le trait, les premières années de la présence de l'organisation communautaire sont analysées soit comme une contribution pour rapprocher les services publics des besoins des communautés - c'est l'approche communautaire selon la Fédération des CLSC du Québec (FCLSCQ, 1994) -, soit comme une pratique de solidarité avec les classes populaires contre un État capitaliste - c'est l'approche du Regroupement des organisateurs communautaires du Québec (ROCQ, 1979).

En prenant en compte les grands marqueurs historiques des services sociaux au Québec (Mayer, 2002), on peut baliser le parcours de l'organisation communautaire en CLSC et CSSS en trois périodes distinctes. La première est celle de la mise en place des CLSC qui va de l'installation des premiers CLSC en 1972 à l'achèvement du réseau en 1985. Cette première période est aussi celle des premières embauches d'OC dans le secteur public que l'on sélectionnait souvent en tenant compte de leur expérience en action communautaire dans les milieux locaux ou en animation sociale dans les agences de services sociaux. La fonction de liaison des $\mathrm{OC}$ durant cette première période sert à l'ancrage communautaire de ces nouveaux établissements de première ligne. Durant la seconde période qui va de 1986 à l'intégration des CLSC dans les CSSS en 2004, les CLSC suivent un parcours de structuration comme établissements du réseau public et l'organisation communautaire s'organise comme pratique professionnelle. La fonction de liaison des OC se déploie alors dans le développement des communautés et dans les concertations locales des acteurs sociaux et le soutien aux organismes d'action communautaire. La recherche de Comeau et al., (2008), réalisée à la toute fin de la période des CLSC, reprend les questionnements de la recherche de Hurtubise et al. (1989) et permet de mesurer l'évolution des pratiques en termes d'autonomie professionnelle. Elle confirme que l'évolution des pratiques d'organisation communautaire en CLSC ne s'est pas faite aux dépens de l'autonomie professionnelle des OC. La dernière période s'ouvre avec la création des CSSS en 2004 et la disparition des CLSC comme établissements autonomes dans le cadre d'un processus rapide de regroupement des établissements de santé et de services sociaux. Cette transformation institutionnelle place l'organisation communautaire dans une situation relativement complexe: une conjoncture sociale marquée par la nouvelle gestion publique (Merrien, 1999); un territoire d'intervention pour lequel le CSSS doit assumer la responsabilité populationnelle, élargie en fonction des services hospitaliers ; et la multiplication des approches programmées. Dans l'exercice de leur fonction de liaison, les OC doivent composer avec une nouvelle réalité institutionnelle et des politiques plus centralisatrices qu'au cours des périodes précédentes.

La littérature, qui aborde la liaison, la présente davantage comme une stratégie des organismes de service que comme fonction des OC. Taylor (1985) propose la Community Liaison Approach visant à mettre un organisme de services en lien avec son milieu d'intervention ce qui donne à l'organisation communautaire une portée institutionnelle et politique. Ce point de vue est repris par Weil (2005) pour souligner la pertinence d'associer les acteurs d'une communauté à la définition des services. Kurzman (1985) de même que Stoner (1985) identifient, pour leur part, des habiletés stratégiques et des savoir-faire techniques qui 
sont requis pour soutenir ces liaisons. Du côté français, on s'intéresse aux tâches de traduction favorisant la concertation (Beuret, 2006), un concept relativement proche de la liaison. Cependant ces auteurs n'abordent pas la pratique de ceux et celles qui assument la fonction de liaison sur une base professionnelle. C'est dans les récits de pratique que la liaison est identifiée par des praticiens comme une fonction des OC (RQIIAC, 2010, p. 102 ; Lachapelle, Bourque et Foisy, 2009).

La question se pose donc de la fonction de liaison des OC dans le contexte actuel caractérisé par une très forte sollicitation des communautés et du mouvement communautaire par les divers programmes qui descendent dans les collectivités (Santé publique, sociétés de gestion issues de la Fondation Lucie-et-André-Chagnon, Plan d'action gouvernemental pour la solidarité et l'inclusion sociale, etc.), la tendance à la centralisation des politiques publiques et à l'utilisation des ressources locales comme complément de l'action publique, et la responsabilité populationnelle de CSSS organisés autour des hôpitaux et soumis à des pressions pour de nouvelles fusions d'établissements. Les OC peuvent-ils encore exercer cette fonction dans les CSSS et les communautés locales ?

\section{Considérations méthodologiques}

La recherche a été menée selon les principes de la théorisation enracinée (grounded theory) (Luckerhoff et Guillemette, 2012 ; Strauss et Corbin, 1998), c'est-à-dire en identifiant à partir des informations recueillies ce qui renvoie à la fonction de liaison plutôt qu'en partant d'une définition à vérifier dans le cadre de la collecte de données. Ce choix s'appuie sur la théorie de la structuration de Giddens (1984), selon laquelle les principes structurels créent des configurations institutionnelles bien identifiables à la fois dans l'espace et dans le temps. Ces principes structurels conditionnent les pratiques des acteurs en même temps qu'ils sont influencés par ces mêmes pratiques. Les contraintes du cadre institutionnel, les acteurs en ont une conscience pratique qu'ils ne peuvent pas nécessairement expliciter, mais qui leur permet néanmoins d'avoir des pratiques qui contribuent au changement social. Ils ont aussi une intelligence pratique qui se traduit dans des routines repérables et dans le discours qu'ils tiennent sur leur action. Ce sont ces routines liées à la fonction de liaison que nous avons cherché à identifier dans le discours des personnes qui nous ont accordé une entrevue.

Vingt-deux entrevues individuelles semi-dirigées ont été réalisées dans huit systèmes locaux d'action (SLA) différents d'une même région administrative du Québec. Ces SLA, dont l'intérêt tient à ce qu'ils sont des espaces d'appartenance, correspondent généralement à un territoire de CLSC ou de municipalité régionale de comté (MRC). Les informatrices et informateurs ont été d'abord un ou une OC par SLA à qui nous avons demandé de nous accorder une entrevue. Huit ont répondu positivement. Suite à l'entrevue, il a été demandé à chacun de nous proposer deux autres personnes à rencontrer pour mieux comprendre leur pratique. Quatorze des seize personnes proposées ont accordé une entrevue. Les 22 entrevues ont donné la parole à 8 OC et 5 agents de développement rural (ADR) dont les pratiques comportent la mise en œuvre d'une fonction de liaison, et à 9 autres informatrices et informateurs avec lesquels les premiers interagissent dans leurs pratiques. S'est ajoutée une entrevue de groupe avec 5 OC retraités de la même région afin de placer dans une perspective historique l'évolution des pratiques de 
liaison. Enregistrées numériquement et transcrites intégralement, ces entrevues ont fait l'objet d'un codage réalisé de manière inductive de telle sorte que les résultats de la recherche reflètent le plus fidèlement possible le discours des $\mathrm{OC}$ et autres agents de liaison sur la mise en œuvre de la fonction de liaison dans les SLA où ils interviennent.

\section{Une fonction marquée par l'évolution du contexte}

Les caractéristiques structurelles de chaque période ont conditionné les pratiques de liaison. L'organisation communautaire durant la période de mise en place des CLSC a soutenu l'engagement de ces nouveaux établissements dans le développement de relations avec les populations et les organismes du milieu local. Ces rapports ont été parfois marqués par la collaboration, parfois tendus, mais ils correspondaient à un choix institutionnel clair généralement favorable aux pratiques de liaison avec le milieu, comme l'exprimait un OC : «On sentait qu'on avait une organisation qui était complice de l'action que l'on faisait. » Les entrevues ont donné de cette période un portrait qui correspond bien aux résultats des recherches antérieures quant à la contribution de l'action communautaire au caractère innovateur des CLSC (NPS, 1988 ; Favreau et Hurtubise, 1993 ; Beauchamp, 2006).

À propos de la période d'institutionnalisation des CLSC et de reconnaissance de l'action communautaire autonome, les OC soulignent l'investissement dans le développement des communautés comme caractéristique de la fonction de liaison. Par exemple, pour mettre en œuvre la politique de santé mentale, il fallait « mettre ensemble les organismes communautaires, la psychiatrie, les CLSC, les ressources de réadaptation autour de ce qu'ils appelaient les comités tripartites». Ces approches supposent de savoir «additionner les forces, savoir négocier et faire accepter les désaccords sans tomber dans les affrontements stériles » (Favreau et Hurtubise, 1993, p. 80). Dans ces concertations, initiées en grande majorité à partir du milieu local (Côté, St-Louis et Bourque, 2010), les pratiques de liaison visaient à associer pouvoirs locaux et action citoyenne dans le contexte de la promotion du développement social (CSBE, 1998) et de la reconnaissance par l'État de l'économie sociale (Chantier de l'économie sociale, 1996) et de l'action communautaire (Gouvernement du Québec, 2001).

Depuis l'avènement des CSSS, l'expertise acquise par les OC est confrontée à une modification substantielle de l'espace dans lequel elle doit se déployer: modification des territoires institutionnels, notamment avec les fusions municipales en 2000 et le regroupement de plusieurs territoires de CLSC en 2003 ; multiplication des démarches de concertation le plus souvent initiées de l'extérieur des milieux, selon une logique descendante avec ses effets d'hyperconcertation (Bourque, 2008) ; déconcentration au niveau des Conférences régionales des élus (CRÉ) et des MRC des programmes de l'État québécois et des sociétés en partenariat avec la Fondation Lucie-et-André-Chagnon, confiant aux élus de nouvelles charges quant au développement social et à l'occupation des territoires; multiplication des agents de développement dédiés au soutien des diverses concertations locales et régionales. Les OC des CSSS sont à l'emploi d'une institution dont la mission est définie par la responsabilité sur le plan sociosanitaire de la population d'un territoire, alors que l'occupation et le développement de ces territoires, de même que la solidarité et l'inclusion sociale, sont confiés aux MRC et aux CRÉ, et que l'éducation demeure un réseau dont la gestion est très fortement centralisée à Québec. Le 
développement des communautés comme stratégie de santé publique offre un pont entre les CSSS et les MRC-Centres locaux de développement (CLD), mais sa place parmi les programmes-services des CSSS demeure souvent marginale en comparaison de ce que permettaient des CLSC autonomes en termes de rapports aux communautés. Ces changements soulèvent des inquiétudes chez les OC maintenant à la retraite, quant à la marge d'autonomie professionnelle de la nouvelle génération, une marge dont eux-mêmes bénéficiaient du fait de leur présence historique dans les établissements, de leurs liens avec la direction et du travail en équipe d'OC.

Le contexte institutionnel des CSSS remet à l'ordre du jour des questions qui ont marqué I'histoire des CLSC depuis leurs débuts en ce qui concerne les relations des OC avec leur établissement: faut-il, comme le formulait le Rapport Brunet (Brunet et al., 1987), que l'organisation communautaire se centre uniquement sur les programmes du réseau de santé et de services sociaux ou bien peut-elle aussi se définir à partir des enjeux des communautés dans lesquelles le CSSS dispense ses services ? Les trois directions qui nous ont accordé une entrevue, tout en reconnaissant l'importance de l'ancrage des OC dans le milieu, ont soulevé des questions sur leurs mandats axés vers l'extérieur et exprimé la préoccupation que les OC orientent davantage leurs pratiques vers les services du CSSS de façon à contribuer à leur « communautarisation ». Déjà au début des années 1990, Favreau et Hurtubise remarquaient la « place stratégique des organisateurs communautaires dans la mission communautaire des CLSC » (Favreau et Hurtubise, 1993, p. 169), ajoutant que cela « ne réussit que là où les organisateurs communautaires y mettent du leur » (ibid., p. 170), notamment parce qu'ils ont une présence significative dans le milieu. Pour les OC des CSSS, l'alternative entre intervenir dans le milieu ou en établissement n'en est pas une. Entre la réponse aux attentes de directions de programme qui souhaitent que le milieu soit mobilisé en regard de leurs services, et la réponse aux attentes du milieu en termes de développement des communautés, privilégier l'action à l'intérieur du CSSS serait, comme le dit un OC, jouer « la mobilisation sociale versus le développement des communautés », alors qu'un vrai partenariat repose sur des relations qui respectent chacun des partenaires. Établir la liaison, c'est faire en sorte que, de part et d'autre, on connaisse et prenne en compte les différents points de vue. À cet égard, au moment de notre recherche, les jeux n'étaient pas encore faits: "Je dirais qu'ici le Centre de santé est à la recherche d'un point d'équilibre » entre ces deux pôles de l'approche communautaire, conclut cet OC.

D'un point de vue historique, on peut parler à la fois d'une certaine pérennité puisque, dans la complexité institutionnelle de la période actuelle, les OC exercent toujours la fonction de liaison, et d'une évolution de cette fonction depuis les pratiques de développement de groupes communautaires et d'ajustement des services, caractéristiques des débuts des CLSC, jusqu'au déploiement de pratiques complexes en fonction du profil de chaque système local d'action (SLA) où les CSSS sont présents à divers degrés.

\section{Une fonction mise en œuvre selon les SLA}

La recherche a montré que l'exercice de la fonction de liaison en organisation communautaire est maintenant partagée avec d'autres intervenantes et intervenants en développement 
d'actions territorialisées. La fonction de liaison apparaît ainsi comme une pratique de soutien à la territorialité qui sera décrite dans la première partie de cette section. Elle apparaît aussi comme une fonction axée sur la participation citoyenne, un trait commun aux praticiennes et praticiens du développement qui sera abordée dans la deuxième partie de la section.

L'actualité de la fonction de liaison se vérifie dans les pratiques d'organisation communautaire qui caractérisent un ensemble de métiers reliés au développement des territoires : OC en CSSS, ADR des CLD, agents de projet, etc. Tous les OC rencontrés dans la recherche ont identifié des pratiques de liaison avec des organismes de leur milieu d'intervention: ils sont partie prenante non seulement du réseau qui les emploie et de ses préoccupations, mais aussi d'un ou plusieurs SLA déterminés par les acteurs du milieu. Dans les SLA, le leadership institutionnel relève des directions des institutions et organismes qui assument des missions sectorielles. À l'échelle locale, sur le terrain économique les CLD, les corporations de développement économique communautaire (CDEC) et les sociétés d'aide au développement des collectivités (SADC) exercent une mission définie par des programmes publics et disposent de ressources publiques pour soutenir une planification stratégique locale. Sur le terrain de l'éducation et de la formation, les commissions scolaires et les cégeps, mais aussi les centres locaux d'emploi (CLE) et les carrefours jeunesse emploi (CJE) sont des instances qui apportent aux collectivités des ressources essentielles. Les CSSS dispensent, en première ligne, neuf grands programmes de services sociaux et de santé et agissent comme porte d'accès aux services spécialisés de deuxième ligne. Le leadership citoyen passe notamment par les organismes d'action communautaire, souvent regroupés sur le plan territorial en corporation de développement communautaire (CDC).

La fonction de liaison se présente d'abord comme un soutien à la territorialité (Caillouette et al., 2007) et les OC l'exercent en soutien à la participation citoyenne dans les SLA qui sont des espaces de rencontre des logiques descendante ou de programme et ascendante ou citoyenne. Elle s'exerce aussi dans les rapports avec le palier régional - via les CRÉ, les agences de la santé et des services sociaux (ASSS) et les conférences administratives régionales (CAR) auquel l'État confie actuellement la gestion des programmes publics et les ressources d'expertise aussi bien que les moyens financiers dont les SLA ont besoin pour mettre en œuvre leurs plans d'action.

\subsection{La fonction de liaison en soutien à la territorialité}

Les SLA sont constitués par les divers acteurs d'un milieu donné et sont des espaces où se rencontrent, d'une part, les projets, initiatives et demandes, issues de la communauté, et, d'autre part, des programmes publics qui transitent généralement par des instances régionales. Les OC assument une fonction de liaison entre ces diverses logiques dans le cadre de démarches de concertation et de projets intersectoriels. L'étude des caractéristiques territoriales, qui déterminent la mise en œuvre de la fonction de liaison, a montré qu'elles ont peu à voir avec la situation socioéconomique d'un territoire et qu'elles relèvent davantage de la territorialité vécue et, surtout, de la place que leur accordent les autorités locales. Les directions des institutions sont, à cet égard, les acteurs les plus directement concernés. 
Les démarches vécues dans 3 SLA ont produit des résultats qui modifient la capacité des acteurs d'agir sur le développement de leur territoire et leur accès aux ressources pour mettre en œuvre leurs projets. Ces démarches ont rendu possibles des réalisations concrètes en termes d'occupation et de développement du territoire. Les OC et ADR, qui ont animé ces démarches, ont traduit en termes accessibles les connaissances expertes développées par les institutions et pris le temps requis pour que les acteurs locaux se les approprient. Ils ont aussi établi le rythme des travaux de façon que se créent des liens entre les acteurs concernés par la situation, et pour que ceux-ci soient en mesure de définir un projet répondant à leur perception des problèmes et enjeux de leur milieu. Les SLA ainsi constitués ont été en mesure de décrocher l'appui d'instances régionales et nationales qui leur ont donné accès à de nouvelles ressources. Les OC et ADR d'un second groupe de 2 SLA ont été, quant à eux, en mesure d'intervenir à l'échelle régionale pour que la mise en œuvre du Plan d'action gouvernemental pour la solidarité et l'inclusion sociale (PAGSIS) fournisse des ressources qui répondent aux besoins des divers SLA engagés dans la lutte contre la pauvreté et pour l'inclusion et la solidarité sociale.

Les agents de liaison de ces cinq premiers territoires, OC et ADR, ont eu toute la latitude requise pour mettre en œuvre leur expertise, n'étant contraints ni par des impératifs du CSSS, employeur des OC, ni par ceux du CLD ou de la MRC, employeur des ADR. Cette marge de manœuvre est par ailleurs actuellement remise en question par des directions de CSSS qui manifestent une volonté de la baliser en fonction d'une certaine conception de la responsabilité populationnelle qui leur est confiée.

Les OC d'un troisième groupe de 2 SLA se trouvent, quant à eux, dans des situations où ils reçoivent peu d'appui des autorités locales. Cela ne les empêche pas de collaborer avec des acteurs locaux, mettant en œuvre des pratiques de liaison à des échelles plus restreintes, dans le cadre de comités municipaux de développement ou de projets locaux dont la portée, en termes de développement des communautés, est moins structurante que dans le premier groupe. Leurs relations avec les directions sont empreintes d'ambigüité, voire de tensions qui sont perçues par les autres acteurs du milieu. Enfin un dernier SLA n'avait pas de soutien en organisation communautaire du CSSS au moment de la collecte de données car les postes d'OC étaient dépourvus de titulaire. C'est le milieu communautaire qui assurait la liaison pour la mise en œuvre du PAGSIS.

Les rapports des OC avec les élus ont évolué au fil de l'histoire des CLSC et des CSSS. Dans la phase d'implantation des CLSC, la proximité des OC avec leur direction leur donnait une bonne marge d'autonomie professionnelle dans les démarches avec des élus qui, à cette époque, se sentaient en général relativement peu concernés par les enjeux sociocommunautaires. Le travail de liaison passait beaucoup par la sensibilisation des élus aux enjeux sociaux. Par la suite, dans les CLSC en phase d'institutionnalisation, les OC bénéficiaient de la même légitimité politique que les autres acteurs de l'action communautaire: leurs relations avec les élus évoluaient à la mesure des politiques d'un État qui se montrait favorable aux partenariats avec la société civile, ce qui souvent entrainait des résistances du côté des élus. Avec l'adoption en 2003 de la loi créant les CRÉ et soumettant les CLD aux MRC, les élus se trouvent dans une nouvelle position: l'État met en place des programmes 
accompagnés de ressources, dont il leur confie le contrôle. Ils sont des acteurs de pouvoir dans les SLA. Leur adhésion ou non aux démarches décide souvent de la réalisation ou non des projets. Ils ne sont cependant pas en rapport hiérarchique direct avec les OC ni les ADR qui relèvent d'une direction institutionnelle. Ils font partie des acteurs à mettre en liens dans le cadre des SLA. Les agents de liaison, OC et ADR, ont intérêt à bien les connaître et à développer un sens politique pour comprendre comment les associer aux projets du territoire.

La fonction de liaison est donc mise en œuvre dans le cadre de SLA très diversifiés qui donnent lieu à des rapports complexes au pouvoir (Comeau, 2007 ; Bourque, 2008 ; Klein, 2011). Le développement de la territorialité, de l'appartenance au territoire constitue un enjeu majeur pour les collectivités locales soumises à l'effet déstructurant du mode actuel de régulation libéral productiviste (Lipietz, 2012 ; Weil, 2005). L'engagement des établissements, les profils des dirigeants et le leadership politique aussi bien que les traits culturels de chaque milieu conditionnent le fonctionnement des SLA. Dans ce contexte, la contribution des praticiens de la liaison passe par la mise en œuvre d'« habiletés stratégiques » que Kurzman identifiait à la capacité de « jauger les intérêts et engagements des parties » et à « entrevoir les possibilités au-delà des évidences » (notre traduction de Kurzman, 1985, p. 109) de façon à permettre «à chacun de construire son point de vue pour participer à la construction d'un point de vue collectif »(Beuret, 2006, p. 46). On peut considérer que les SLA affichant des réalisations structurantes ont connu une gestion efficace de ces rapports au pouvoir.

\subsection{Les OC professionnellement dédiés à la participation citoyenne}

La préoccupation de favoriser la participation des populations est une constante des entrevues réalisées avec les OC, les ADR et les personnes intervenant dans le milieu communautaire. On a mentionné plusieurs fois l'importance d'inclure les personnes vulnérables dans les démarches de développement social et de lutte contre la pauvreté. II n'entrait pas dans les perspectives de la recherche de vérifier le niveau de concrétisation sur le terrain de cette visée, mais une part significative des efforts de liaison va aux démarches qui donnent la parole aux citoyennes et citoyens dans les comités de développement local créés dans le cadre du Pacte rural, dans les instances où se fait le choix des priorités pour le PAGSIS, dans la mise en œuvre des projets de revitalisation des secteurs défavorisés des MRC, etc. Ces efforts ne se limitent pas à des techniques d'animation. Ils comportent un travail d'accompagnement visant à développer les capacités et le leadership des personnes participantes, aussi bien dans les projets de développement dans leur propre milieu que dans les instances de représentation à l'échelle régionale: «On s'est donné plusieurs étapes et un outil qui permet de garder la parole citoyenne. » Le test d'une liaison réussie, c'est de voir des citoyennes et citoyens prendre en charge les diverses dimensions de l'action.

Cet enjeu de participation établit une ligne de démarcation entre les approches instrumentalisantes de planning social (Rothman et Tropman, 1987) et celles qui favorisent l'appropriation comme l'approche socio-institutionnelle (Bourque et Lachapelle, 2007). Le soutien à la participation citoyenne représente la plus-value de l'organisation communautaire par rapport aux démarches de prévention-promotion et de marketing social visant à implanter des programmes. En ouvrant les SLA aux acteurs qui ont généralement peu ou pas d'accès au 
pouvoir, l'organisation communautaire favorise l'établissement de rapports plus symétriques (Proulx, Bourque et Savard, 2005) dans les processus d'hybridation des logiques descendante et ascendante. Cela représente un gain pour tous puisque l'efficacité des programmes est d'autant plus grande qu'ils accordent une priorité aux enjeux locaux et à la participation citoyenne (Bilodeau et al., 2004).

La capacité des OC de contribuer aussi bien au développement des communautés qu'aux programmes du CSSS repose sur leur connaissance relationnelle du milieu : " s'imbiber de ce que le groupe vit, de ce que le quartier vit» (Lachapelle, Bourque et Foisy, 2009, p. 36). En s'inscrivant dans les SLA et en y exerçant une fonction de liaison, ils ne sont pas à l'extérieur du CSSS, ils interviennent selon leur compétence professionnelle. C'est en étant présents dans les milieux qu'ils développent une expertise utile pour les approches communautaires dans les services, d'abord en termes de connaissance du milieu auquel ils sont destinés, mais aussi en termes de capacité de mettre les intervenantes et intervenants du CSSS en relations avec les acteurs du milieu. Leur réseau de relations est une dimension de la fonction de liaison dont les programmes-services ont besoin pour assumer leur responsabilité populationnelle.

\section{Les actions de la liaison}

Au terme de cette analyse de pratiques d'OC et d'ADR, la liaison comme fonction en organisation communautaire se présente comme un ensemble d'actions permettant de mettre en lien les acteurs d'un SLA, d'accroître leur capacité collective d'agir et de produire des résultats: connaître le milieu, mobiliser les acteurs, démocratiser les rapports au pouvoir, garantir la cohésion du SLA et rendre accessibles les ressources.

La connaissance des données statistiques et des profils de chaque territoire fait partie des compétences des OC et des ADR, mais on apprécie surtout leur capacité de présence : « L'OC sent bien, il "piffe" bien quand il y a quelque chose qui se passe et il arrive au moment où cela se produit », affirme une direction de CSSS. Ils se distinguent par leur familiarité avec le milieu, leur connaissance des liens entre les personnes et les organismes: "II est connu de tout le monde, affirme une direction, il est très, très présent dans la communauté. » Cette connaissance relationnelle et dynamique est le fruit de la durée de présence dans le milieu et de la carte de relations établie par l'OC ou l'ADR. Cela se traduit par l'expression « être proche ».

Mobiliser les acteurs c'est d'abord établir des liens avec eux et entre eux : « L'OC amène les gens à réfléchir à ce que, pour pouvoir travailler ensemble, il faut d'abord se connaître et se reconnaître », considère un ADR. C'est accompagner le SLA dans une démarche d'actionréflexion-action de façon à développer des leaders et une intelligence collective au service du milieu : «Le groupe de partenaires qui est impliqué dans cette démarche-là, ils sont allés en France avec les gens du milieu pour leur montrer. » Mobiliser, c'est miser sur les forces du milieu pour lui permettre de développer son autonomie d'action : «C'est beaucoup, affirme une $\mathrm{OC}$, les amener à réfléchir et voir c'est quoi leurs forces, qu'est-ce qu'ils peuvent faire. » Autrement exprimé par une ADR : «Tu le fais avec les gens et tu peux comprendre [...] qui est en mesure de "leader", qui est en mesure de mener un projet. » Cela se traduit bien dans l'expression « faire avec ». 
Garantir la cohésion du SLA, c'est mettre à contribution tout ce qui peut tisser et renforcer les liens entre les acteurs, élargir leurs réseaux de relations et les rendre conscients de leurs forces: "On a fait comprendre aux maires tranquillement pas vite que si on travaille tout le monde à améliorer la qualité de vie on va améliorer aussi les conditions de vie des gens », dit un OC. Cela exige d'assurer la transparence et la circulation de l'information et d'entretenir entre les acteurs les conditions du dialogue, y compris dans les situations de conflit, en posant au besoin des gestes de médiation. Bref, garantir la cohésion, c'est « être fiable ».

Démocratiser les rapports au pouvoir, c'est favoriser la participation des citoyens et citoyennes, y compris de ceux et celles qui sont sans pouvoir. Voici comment un OC présente ce type d'action: «C'est comme si l'agent de liaison, pour moi, devait avoir deux bras : il y a le bras qui accueille la demande et il y a le bras qui dit, regarde, il y a une communauté. On va le faire ensemble. [...] II y aura pour moi toujours nécessité de m'assurer que la parole citoyenne est entendue. » Pour y arriver, il faut développer des démarches inclusives, mais aussi la capacité d'agir des gens et accepter de vivre les rapports de force par lesquels passe le renforcement de la capacité démocratique des SLA. Pour y arriver il faut «avoir un sens politique ».

Rendre accessibles les ressources, c'est mobiliser les ressources locales et donner accès aux ressources externes requises pour que le SLA réalise ses projets. Deux expériences de SLA sont à cet égard éloquentes : l'engagement des acteurs locaux a permis de décrocher des ressources financières importantes qui leur ont permis de réaliser des projets qu'ils n'auraient pas envisagé entreprendre auparavant. C'est finalement le test de la fonction de liaison: amener un SLA à produire des résultats. L'organisation communautaire doit « être efficace ».

Mettre en œuvre la fonction de liaison, c'est donc être proche, faire avec, être fiable, avoir un sens politique et être efficace. C'est ce qui permet aux divers leaderships constitutifs d'un SLA de travailler de façon concertée. Exercer la fonction de liaison, c'est assumer un leadership de processus au service à la fois des leaderships de pouvoir, institutionnel et politique, et des leaderships de participation, citoyen et communautaire. Être passeur, c'est faire en sorte que tous les acteurs aient la possibilité d'apporter leur contribution dans le cadre de SLA efficaces. Les OC des CSSS ne remplissent pas à eux seuls toutes les fonctions de liaison, mais tous mettent en œuvre les dimensions constitutives de cette fonction.

\section{Les conditions d'exercice dans le contexte actuel des CSSS}

Les résultats de cette recherche rejoignent la conclusion d'une récente démarche de recherche action menée conjointement en France et au Québec: «De plus en plus, l'apport des professionnels du développement territorial est de permettre les transactions entre les acteurs différents, jouant un rôle essentiel de médiation, d'intermédiation, de traduction, [...] d'interface. » (Vincent et Kellenberger, 2013, p. 14). Ces conclusions confirment l'actualité de la fonction de liaison face aux enjeux des pratiques d'organisation communautaire, non seulement en CSSS, mais aussi dans un ensemble de métiers reliés au développement des territoires. Les OC partagent avec les autres praticiennes et praticiens du développement territorial une expertise d'une particulière actualité. 
La fonction de liaison dépasse une simple habileté professionnelle. Elle se présente toujours en fonction du contexte dans lequel elle s'exerce comme pratique de mise en interrelation d'acteurs collectifs. Sa pertinence repose sur deux enjeux auxquels sont actuellement confrontées les collectivités locales: la redéfinition de la territorialité, à la fois comme appartenance et comme mode d'occupation du territoire, et la participation citoyenne dans une société où l'exercice de la démocratie est mis à l'épreuve. Les pratiques de liaison exigent une durée de présence permettant de bien connaître les acteurs locaux et d'être reconnu comme partenaire fiable, une volonté de soutenir l'action des acteurs locaux et une capacité de mobiliser leurs ressources locales et de donner au milieu un accès aux ressources externes.

La recherche a aussi mis en évidence l'importance des conditions d'exercice de la fonction de liaison. Dans les CSSS, la conception de la responsabilité populationnelle véhiculée par la direction constitue le contexte politique des pratiques de liaison : les établissements ont tout à gagner à miser sur la capacité des OC à susciter et à soutenir la capacité d'innovation des milieux. Pour y arriver, ceux-ci doivent avoir la possibilité d'assurer une présence dans le milieu significative en termes de temps et jouir d'une marge d'autonomie professionnelle. Les directions des CSSS doivent aussi reconnaître que les OC ont besoin d'une communauté de pratique pour maintenir et développer les expertises complexes sur lesquelles repose la fonction de liaison. Ces enjeux prennent un relief particulier dans le contexte actuel où se posent des défis de développement durable des communautés, de contraintes budgétaires et de remise en question du modèle québécois de partenariat entre l'État et la société civile.

LACHAPELLE, René

Professionnel de recherche

Chaire de recherche du Canada en organisation communautaire Université du Québec en Outaouais

\section{BIBLIOGRAPHIE}

Beauchamp, Gilles (2006). Entre l'institution et la communauté, des transactions aux frontières. 1975-1990: quinze années de pratiques d'organisation communautaire au CLSC Hochelaga-Maisonneuve, version modifiée du mémoire de sociologie présenté comme exigence partielle à l'obtention d'une maîtrise à l'Université du Québec à Montréal en 1991, $62 \mathrm{p}$.

Beuret, Jean-Eudes (2006). La conduite de la concertation. Pour la gestion de l'environnement et partage des ressources, Paris, L'Harmattan, $340 \mathrm{p}$.

Beuret, Jean-Eudes (2010). « De la négociation conflictuelle à la négociation concertative : un "point de passage transactionnel" », Négociations, n² 13, De Boeck Université, p. 43-60.

Bilodeau, A., D. Allard, D. Francoeur et P. Chabot (2004). « L'exigence démocratique de la planification participative: le cas de la santé publique au Québec », dans Nouvelles pratiques sociales, vol. 17, $\mathrm{n}^{\circ} 1$, p. 50-65.

Bourque, Denis (2008). Concertation et partenariat. Entre levier et piège du développement des communautés, Québec, Presses de l'Université du Québec, 142 p. 
Bourque, Denis, et René Lachapelle (2007). « L'approche socio-institutionnelle en organisation communautaire » in Bourque, Denis, Yvan Comeau, Louis Favreau et Lucie Fréchette (dir.), L'organisation communautaire. Fondements, approches et champs de pratique, Québec, Presses de l'Université du Québec, p. 101-117.

Brunet, Jacques, et al. (1987). Rapport du Comité de réflexion et d'analyse des services dispensés par les CLSC, $92 \mathrm{p}$.

Caillouette, Jacques, Nicole Dallaire, Ginette Boyer et Suzanne Garon (2007). «Territorialité, action publique et développement des communautés », Économie et Solidarité, vol. 38, $\mathrm{n}^{\circ} 1$, p. 8-23.

Chantier de l'économie sociale (1996). Osons la solidarité !, Rapport du groupe de travail sur l'économie sociale, Sommet sur l'économie et l'emploi, 64 p.

Comeau, Yvan (2007), « L'approche de développement local en organisation communautaire », in Bourque, Denis, Yvan Comeau, Louis Favreau et Lucie Fréchette (dir.), L'organisation communautaire, fondements, approches et champs de pratique, Québec, Presses de I'Université du Québec, p. 59-79.

Comeau, Yvan, Martine Duperré, Yves Hurtubise, Clément Mercier et Daniel Turcotte (2008), L'organisation communautaire en mutation. Étude sur la structuration d'une profession du social, Les Presses de l'Université Laval, 194p.

Côté, Nathalie, Marie-Pier St-Louis et Denis Bourque (2010). Pratiques de concertation sur le territoire de la MRC des Moulins : rapport de la démarche de recherche participative, Chaire de recherche du Canada en organisation communautaire, Cahier $n^{0} 1003,78 \mathrm{p}$.

CSBE (Conseil de la santé et du bien-être), (1998). Forum sur le développement social, Rapport, Rapport remis au ministre de la Santé et des Services sociaux, 140 p.

Doré, Gérald, et Claude Larose (1979). «L'organisation communautaire: pratique salariée d'animation des collectivités au Québec », Service social, vol. 28 n ${ }^{\text {os }} 2-3$, p. 210-230.

Favreau, Louis, et Yves Hurtubise (1993). CLSC et communautés locales. La contribution de l'organisation communautaire, Sainte-Foy, Presses de l'Université du Québec, 211 p.

FCLSCQ (Fédération des CLSC du Québec) (1994). Pratiques d'action communautaire en CLSC, Document de réflexion, $92 \mathrm{p}$.

Giddens, Anthony (1984). The Constitution of Society. Outline of the Theory of Structuration, Berkeley and Los Angeles, University of California Press, $402 \mathrm{p}$.

Gouvernement du Québec 2001). L'action communautaire, une contribution essentielle à l'exercice de la citoyenneté et au développement social du Québec, Québec, 60 p.

Gulati, Padi, et Geoffrey Guest (1990). «The Community-Centered Model: A Garden-Variety Approach or a Radical Transformation of Community Practice? », Social Work, vol. 35, $\mathrm{n}^{\circ} 1$, p. 63-68. 
Hurtubise, Yves, Gilles Beauchamp, Louis Favreau et Danielle Fournier (1989). Pratiques d'organisation et de travail communautaires en CLSC, Montréal, RQIIAC (Regroupement québécois des intervenantes et intervenants en action communautaire), $92 \mathrm{p}$.

Jobin, Marie (1986). L'action communautaire dans les CLSC. État de la question, Fédération des CLSC du Québec, 18 p. + Annexes.

Klein, Juan-Luis (2011), «Économie sociale et territoire en contexte de mondialisation: le développement par l'initiative locale » in Bellemare, Guy, et Juan-Luis Klein (dir.). Innovation sociale et territoire. Convergences théoriques et pratiques, Québec, Presses de l'Université du Québec, p. 175-194.

Kurzman, Paul A. (1985). «Program Development and Service Coordination as Components of Community Practice », in Taylor Samuel H. et Robert W. Roberts (dir.), Theory and Practice of Community Social Work, New York, Columbia University Press, p. 95-124.

Lachapelle, René, Denis Bourque, et Dominic Foisy, avec la collaboration de Julie Sénéchal (2009). Apports de l'organisation communautaire en CSSS aux nouvelles infrastructures communautaires de développement des communautés, Rapport de recherche, Chaire de recherche du Canada en organisation communautaire, Université du Québec en Outaouais,, Cahier 09-02, 64 p.

Liedholm, Marianne, et Göran Lindberg (2007). « Un travail de liaison professionnel comme outil d'intégration », Pensée plurielle, De Boeck Université, 2007, n 15, p. 85-90.

Lipietz, Alain (2012). Green Deal. La crise du libéral-productivisme et la réponse écologiste, Paris, La Découverte, 185 p.

Luckerhoff, Jason, et François Guillemette (dir.) (2012). Méthodologie de la théorisation enracinée. Fondements, procédures et usages, Québec, Presses de l'Université du Québec, $282 \mathrm{p}$.

Mayer, Robert (2002). Évolution des pratiques en service social, Boucherville, Gaëtan Morin Éditeur, $489 \mathrm{p}$.

Merrien François-Xavier (1999), «La Nouvelle Gestion publique : un concept mythique », Lien social et Politiques, $n^{\circ} 41$, p. 95-103.

NPS (Nouvelles pratiques sociales) (1988). Les CLSC à la croisée des chemins, vol. 1, $\mathrm{n}^{0} 1$, p. 33-114.

Proulx, Jean, Denis Bourque et Sébastien Savard (2005). Les interfaces entre l'État et le tiers secteur au Québec, Centre d'étude et de recherche en intervention sociale, cahier C-012005, 90 p.

ROCQ (Regroupement des organisateurs communautaires du Québec) (1979). Manifeste du Regroupement des organisateurs communautaires du Québec, $4^{\mathrm{e}}$ édition, polycopié, 38p.

Rothman, Jack, et John E. Tropman (1987). « Models of Community Organization and Macro Practice Perspectives: Their Mixing and Phasing », in Fred M. Cox et al. (dir.), Strategies of Community Organization: Macro Practice, $4^{\mathrm{e}}$ éd., Ithaca (IL), F. E. Peacock Publishers Inc., p. 3-25. 
RQIIAC (Regroupement québécois des intervenantes et intervenants en action communautaire), avec la collaboration de René Lachapelle (2010). Pratiques d'organisation communautaire en CSSS. Cadre de référence du RQIIAC, Québec, Presses de l'Université du Québec, $158 \mathrm{p}$.

Sénéchal, Marcel (1994). «L'action communautaire en CLSC », dans Louis Favreau, René Lachapelle et Lucie Chagnon (dir.). Pratiques d'action communautaire en CLSC. Acquis et défis d'aujourd'hui, Sainte-Foy, Presses de l'Université du Québec, p. 21-23.

Stoner, Madeleine R. (1985). «The Practice of Community Social Work in Mental Health Settings » in Samuel H. Taylor et Robert W. Roberts. Theory and Practice of Community Social Work, New York, Columbia University Press, p. 282-312.

Strauss, Anselm, et Juliet Corbin (1998). Basics of Qualitative Research, Techniques and Procedures for Developing Grounded Theory, Thousands Oaks (CA), Sage Publications, $312 \mathrm{p}$.

Taylor, Samuel H. (1985). «Community Work and Social Work: The Community Liaison Approach », in Taylor Samuel H. et Roberts Robert W. (dir.) (1985). Theory and Practice of Community Social Work, New York, Columbia University Press, p. 179-214.

Vincent, Pascale, et Sonja Kellenberger (2013). Une réflexion sur les enjeux et perspectives pour les métiers du développement territorial, Plateforme des métiers du développement territorial - UNADEL (Union nationale des acteurs et des structures du développement local), $22 \mathrm{p}$.

Weil, Marie (2005). «Introduction, Contexts and Challenges for 21st-Century Communities », in Weil, Marie, Michael Reis, Dorothy N. Gamble, Lorraine Guttiérrez, A. Mulroy et Ram A. Cnaan (dir.) (2005). Handbook of Community Practice, Thousand Oaks (CA), Sage Publications, p. 3-33. 